\title{
Bronzebommeln und Schellen - eine klangliche Errungenschaft aus der Spätbronze- und frühen Eisenzeit
}

\section{Bird-cage bronze pendants and pellet bells - an acoustic achievement of the Late Bronze Age and Early Iron Age}

\author{
Beate Maria Pomberger
}

\begin{abstract}
Abstrakt
Kannte man in der Urgeschichte Europas bis zum Ende des zweiten Jahrtausends Gefäßrasseln aus gebranntem Ton, Holz und getrockneten Früchten, so kommen im 10./9. Jhdt. v. Chr. geschlitzte Bommeln in verschiedensten Formen vom Kaukasus und Nordiran, über Ost- und Mitteleuropa bis zu Ostfrankreich als Anhänger vor. Diese Pendelanhänger gefüllt mit Rasselkörpern ergeben die ersten metallenen Gefäßrasseln (Schellen) und bereichern die Vielfalt der Idiophone mit ihrem hellen "Glockenklang". Die Skythen führten die Erfindung der Schellen als Stangenbekrönungen weiter. Eine eigene Innovation stellen die spätbronzezeitlichen Schellen Mittelirlands dar.
\end{abstract}

\section{Schlagwörter}

Schellen, Schlitzbommeln, Urnenfelderkultur, Hallstattkultur, Spätbronzezeit Irlands, Musikarchäologie, Idiophone, Klang

\begin{abstract}
From the European prehistory until the end of the second millennium BC we know vessel rattles made from fired clay, wood and dried fruits. Then, in the 10th/9th cent. BC, openwork bird-cage bronze pendants came from the Caucasus and North Italy through East and Central Europe to as far as Eastern France. These pendants filled with rattle cores gave rise to the earliest vessel rattles made from metal (pellet bells) and enriched the wide spectrum of idiophones with their bright "sound of bells". The Scythians implemented the invention of jingle bells in their life by mounting them on wooden sticks. A specific innovation represented the Late Bronze Age pellet bells in Central Ireland.
\end{abstract}

\section{Keywords}

pellet bells, openwork bird-cage bronzes, Urnfield Culture, Hallstatt Culture, Late Bronze Age in Ireland, music archaeology, idiophones, sound 


\section{Einleitung}

Im 3. Jahrtausend v. Chr. trat bei den Idiophonen neben dem Klang von Keramik, Holz, Stein, Knochen, Kalkgehäusen von Muscheln und Schnecken, getrockneten Früchten und Kupfer jener der Bronze hinzu. Möglicherweise entdeckte man diesen beim Hämmern von Bronzeblechen, beim mechanischen Nachbearbeiten von gegossenen Bronzeobjekten z.B. dem Ausschmieden von Beilen (Kienlin 2007, 1-22) oder dem Gegeneinanderschlagen zweier Bronzestücke. Der frühbronzezeitliche Schmied kann als erster Entdecker der neuen Klänge gelten, die das menschliche Ohr faszinierten und seitdem nicht mehr aus dem Klangspektrum wegzudenken sind. Sie dominieren auch heute noch neben dem Klang der gespannten Membran das Schlagwerk. Metall besitzt eine etwas längere Nachklangzeit als alle übrigen Materialien der damaligen akustischen Welt. Ihrem hellen silbernen Klang wurden und werden apotropäische Kräfte zugesprochen. Kannte man bis zur Spätbronzezeit tönerne Gefäßrasseln, so entdeckte man in der Zeit des Überganges von der Spätbronzezeit zur frühen Eisenzeit wie man hohle Gefäßkörper mit durchbrochenem Mantel aus Bronze mittels Wachsausschmelzverfahren herstellen kann. Diese Innovation führte in Folge zur „Geburt“ neuer Gefäßrasseln, nämlich der Schellen. Ein weiterer Aspekt besteht darin, dass gleich zwei wichtige Metalle für die Erzeugung von Schellen verwendet wurden: Bronze für den Schallkörper und Eisen für die Rasselkörper. So trifft Eisen auf Bronze im wahrsten Sinn des Wortes. Diese Kombination bestimmt in weiterer Folge auch die meisten Klöppelglocken Europas, die ab der römischen Kaiserzeit in weiten Teilen Verbreitung fanden.

\section{Definition und instrumental- kundliche Einordnung nach der Systematik von Hornbostel und Sachs}

Kugelförmige Pendelanhänger - Bronzebommeln - können massiv oder gitterartig- durchbrochen, innen hohl gegossen werden oder aus Blech geformt werden. Sie haben keinen Rasselkörper in ihrem Inneren. Am oberen Ende sitzt eine Aufhängeöse. Sind sie so angebracht, dass sie gegeneinander oder gegen einen anderen Gegenstand schlagen, können sie zum Klingen gebracht, und als Pendelrassel bezeichnet werden (Systemnummer 112.121).

Gefäßrasseln aus Metall nennt man Schellen oder Rollschellen. Diese werden in der Bronzezeit im Bronzegussverfahren erzeugt. Schellen dienen als Anhänger oder als Stangenkrönung (Zepter). Ihre Füllung besteht aus Eisenkugeln, Bronzekugeln oder Steinchen. Gefäßrasseln (Systemnummer 112.13) zählen zu den mittelbar geschlagenen Idiophonen - Schüttelidiophone -, wobei die Rasselkörper beim Schütteln sowohl gegeneinander als auch gegen die Gefäßwand schlagen (Hornbostel - Sachs 1914, 553-590).

\section{Zur Herstellungsart}

Schlitzbommeln allgemein und durchbrochene Schellen werden mittels Wachsausschmelzverfahren erzeugt. Zunächst müssen für die Schellen geeignete Steinchen gesucht oder die Kügelchen aus Bronze oder Eisen gegossen werden. Diese wiederum werden mit porösem Ton umhüllt und in die gewünschte Kernform gebracht. Über den Kern legt man das gewünschte Bommel/Schellendesign aus einer Schicht Wachs, gestaltet Öse und Eingusstrichter und ummantelt alles mit feinem Lehm. Eine Schicht porösen Tons schützt die Gussform. 
Nach dem Trocknen wird das Wachs vorsichtig ausgeschmolzen, die vorgewärmte Form in ein Sandbett gesetzt und die flüssige Legierung hineingegossen. Der Außenmantel wird in Folge zerschlagen, der Lehmkern vorsichtig herausgekratzt. Zuletzt muss die Oberfläche fein poliert werden (freundliche Mitteilung Clemens Eibner).

\section{Die Klangobjekte}

\subsection{Bronzene Schlitzbommeln}

Bei Schlitzbommeln handelt es sich um runde, ovale oder doppelkonische, innen hohle, gegossene Bronzegegenstände, deren Mantel geschlitzt oder von dreieckigen Öffnungen durchbrochen ist. Fallweise weist die Bommel einen Fuß auf. Am Scheitel sitzt eine einfache, eine erweiterte Öse oder ein figürlicher Aufsatz. Die Verbreitung von Schlitzbommeln reicht von Luristan im Iran, über Armenien, der Kubanregion, Griechenland, Republik Mazedonien, Albanien, Bosnien-Herzegowina, dem Kosovo und Serbien (Bouzek 1974, 278-341; Kilian-Dirlmeier 1979, 78-122; Bouzek 2012, 537-547, Gallus Horvath 1939, Taf. LXXIX) bis hin zu Ostfrankreich. Trachtgegenstände dieser Art datieren um 800 B.C., in die spätgeometrische Zeit, mazedonische Eisenzeit IIA - B, ältere Hallstattkultur HaC (Kilian-Dirlmeier 1979, 98-122, Taf. 31-34; Parzinger - Nekvasil - Barth 1995, 49-55). In Mitteleuropa sowie in Kroatien kommen einige Exemplare bereits in der Phase HaB3 vor (Parzinger - Nekvasil - Barth 1995, 52; VinskiGasparini 1973, 213; Gallus - Horvath 1939).

Kilian-Dirlmeier gliederte die zahlreichen Bommeln aus dem Gebiet Griechenland, Albanien und Mazedonien in konstruktive Elemente (Grundmerkmale) und ergänzende Elemente (Zusatzmerkmale). Sie teilt diese grob zwischen geschlossenen, geschlitzten, durchbrochenen
Bommeln mit vier Stegen und durchbrochenen mit mehr als vier Stegen (Kilian-Dirlmeier 1979, 51, Abb. 1, Abb. 2) ein und unterscheidet darüber hinaus etliche Spielvarianten. In Mitteleuropa kennt man Schlitzbommeln vorwiegend aus der Hallstattkultur - hier vor allem in der Schweiz, Süddeutschland und Ostfrankreich. Sie werden als Schmuckstücke und Teile von Bronzegehängen getragen. Die Bommeln Mitteleuropas und den angrenzenden Gebieten sind etwas einfacher komponiert. Sie kommen hauptsächlich in runder Form mit oder ohne Fuß vor.

Eine geschlitzte Bommel - möglicherweise handelt es sich dabei auch um eine Schelle, was anhand des Bildes und der Beschreibung nicht klar ist - stammt aus dem Urnengräberfeld von Dalj, Kroatien (Vinski-Gasparini 1973, 213 , Tab. 119,6). Zwei geschlitzte hohle Kugeln, die auf Stiften sitzen, wurden in Gajina pedina bei Drežnik, Kroatien, aufgefunden (Vinski-Gasparini 1973 214, Tab. 128, 4, 5). Alle Stücke datieren in die auslaufende Urnenfelderkultur (Phase V bzw. HaB3). Interessant ist, dass Bronzeobjekte mit durchbrochenem Mantel (glockenförmige Anhänger) in Kroatien schon in der Phase II (späte BzD/HaA1) belegt sind (Vinski-Gasparini 1973, 212, Tab. 53, 13, 16).

Aus Slowenien kennt man eine Bronzebommel ohne horizontalen Steg aus Grab 519, Most na Soči, (Teržan - Lo Schiavo - Trampuž-Orel 1984, t. 44 F 519/8; Teržan - Lo Schiavo - Trampuž-Orel 1985, 111, 112). Aus dem ungarischen Gebiet sind vorskythische Funde bekannt: Eine doppelkonische Schlitzbommel aus einem Depot in Ugra, Kom. Bihar, vergesellschaftet mit Pferdezaumzeug und chronolgisch in die Stufe $\mathrm{HaB}$ gestellt, sowie ein Stück aus Pécs (Gallus - Horvath 1939, 18, 50, Taf. XVIII/8, 37, Fig. 3). In derselben Publikation zeigt Tafel VI ein Foto eines geschlitzten Anhängers an einem Bronzeobjekt hängend aus der Sammlung Mauthner, das aus Niederösterreich stammt (Gallus - Horvath 1939, 87, Taf. VI/3). Auf meine Nachfrage 
zum Objekt schrieb mir Ute Luise Dietz Folgendes: „... Der Gegenstand in der Mitte ist singulär. Es kann sich durchaus um einen Teil des Pferdegeschirrs handeln, allerdings nicht in einer praktischen Funktion, sondern als Gehängeteil, z.B. am Joch bzw. der Jochschirrung. Alle Teile gehören zum „thrako-kimmerischen " Material, wobei hiermit m.E. keine ethnische Zuweisung verbunden sein kann...." (Ute Luis Dietz, Universität Frankfurt/Main, Mitteilung vom 13. Juni 2016).

Unbekannt sind der genaue Fundort, die Fundumstände sowie der derzeitige Aufbewahrungsort. Die kleinen auf den Stegen sitzenden Knoten der Bommel weisen auf Ähnlichkeiten mit Stücken aus dem Balkan und Griechenland hin (Kilian-Dirlmeier 1979, Taf. 27/525, Taf. 29/567, 570). Ein Anhänger mit Klapperkettchen und Blechen, gefunden im Brandschüttungsgrab 733, einer Frauenbestattung, das in die Phase HaC (Hodson 1990, 67, 137) datiert, stammt aus Hallstatt-Hochtal, Österreich (Kromer 1959, 151, Taf. 149/1. Gerasselt erklingen Frequenzen ab $5426 \mathrm{~Hz}$ (Pomberger 2016, 138, 264). Aus Treffelsdorf bei Ottmanach, Kärnten, wurde in einem Hort ein Bommelanhänger ausgegraben (Müller-Karpe 1959, 283, Taf. 144/7). Die Westschweiz ist relativ reich an Schlitzbommeln. Es handelt sich dabei um ca. 30 Stück, die Walter Drack in 13 Typen unterteilt, wobei er jedoch zwischen Schellen und Schlitzbommeln keinen Unterschied macht. Ihre Form ist mehr oder weniger kugelig. Sie werden als Anhänger getragen, kommen in Gräbern vor und datieren spätestens in die Stufe HaD1 (Drack 1966, 29-70, Abb. 12, 13).

In Südwestdeutschland sind Schlitzbommeln z. B. in Tauberbischoffsheim-Impfingen, Heidenheim-Schnaitheim (Parzinger - Nekvasil - Barth 1995, 52, Abb.18, 4; Aquarelle von Ludwig Lindenschmit der Ältere in Frey - Nickel - Köster 2009, 107.) nachgewiesen. Zu den Trachtgegenständen der Gruppe Moidons im Jura, gehören ebenfalls geschlitzte Bronzebommeln, länglich oder in Kugelform. Entdeckt wurden sie in Körpergräbern. Ihre Größe liegt zwischen 2-4 cm. Rad- und Bommelanhänger werden öfters zu einem Schmuckstück, befestigt an Textilien, Ketten, Schnüren oder bronzenen Platten, kombiniert getragen (Wamser 1975, 37-65, Taf. 4/1-7; Clodoré 2002, 74, 75, Abb. 38). Ein Schmuckstück mit sieben Bronzebommeln der Variante B an einem Blechband ist aus Clucy (Jura) Frankreich, bekannt und datiert ins Premier Âge de Fer (Clodoré 2002, 74, 75, Abb. 38).

Die „Bommelmode“ erreichte auch die Villanova Kultur der Phasen II und III (800-750 B.C.; 750-680 B.C.) in Oberitalien, wo sie in Gräbern dieser Phase auftreten. Gesellschaftlich hochgestellte Frauen trugen sie zu breiten Bronzegürteln (Müller-Karpe 1959, 220, Abb. 57, $A b b$. 64). Hier sind vor allem die Nekropolen Savena Bologna, Grab 94 (Müller-Karpe 1959, 260, Taf. 79,7-9) - auch: gesehen im Museum Civico Archeologico, Oktober 2014) - und in Benacci Bologna, Grab t. 909 (Poppi - Neri 2015, 38) zu nennen. Das Ensemble eines bronzenen Lendengehänges, aufgefunden auf dem Becken und den Oberschenkeln eines männlichen Skeletts, aus dem hallstattzeitlichen Kultplatz Býčí-skála Höhle bei Brünn, Tschechien, zieren sieben geschlitzte Bronzebommeln mit Klapperblechen. Diese Schlitzbommeln weisen in der Mitte einen leicht hervortretenden umlaufenden Steg auf. Sie werden in die Stufe $\mathrm{HaC} / \mathrm{HaD}$ gestellt (Parzinger - Nekvasil - Barth 1995, 49-55, Abb. 19, 241, Taf. 20, Taf. 69). Zwei Objekte enthielt der Hort von Rataje nad Sázvou, Böhmen, wobei anhand der Fotos nicht klar ist, ob es sich um Bommeln oder Schellen handelt (Šolle 1947/48, 102-104, Abb.1/4 und 12). Ziergehänge mit Bronzebommeln waren auch in Griechenland begehrte Schmuckstücke (Kilian-Dirlmeier 1979, Taf. 74/1310).

Bronzebommeln allgemein fanden als Importgegenstand Eingang in die Lausitzer Kultur/ jüngere Billendorfer Gruppe in Ostdeutschland 

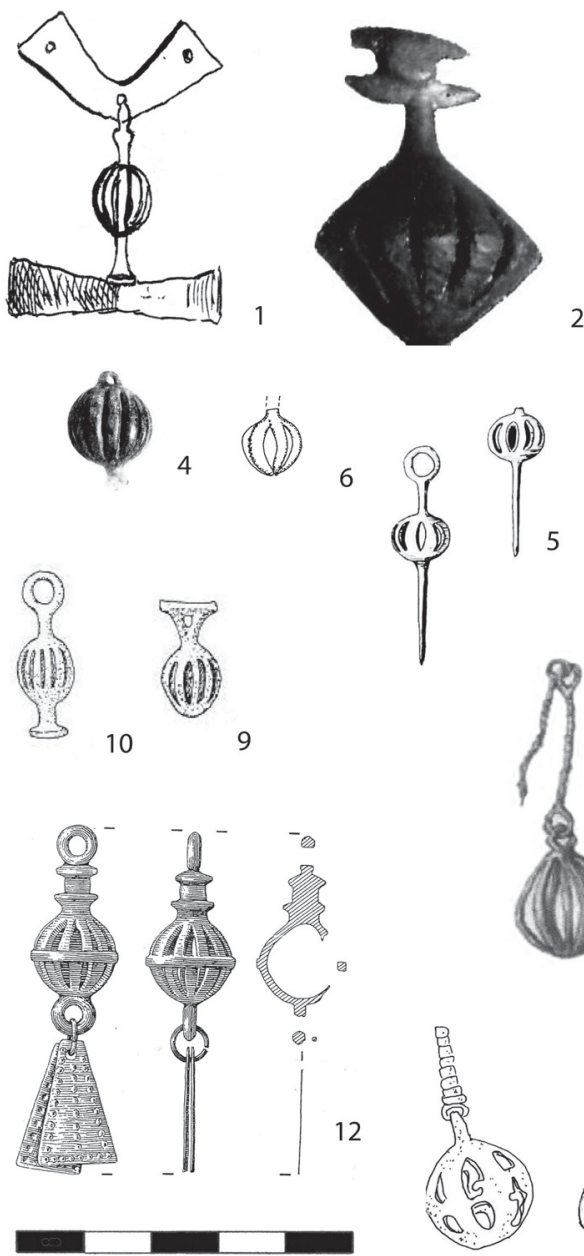

0

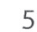

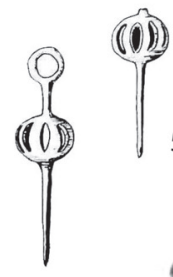

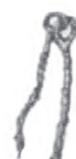

8
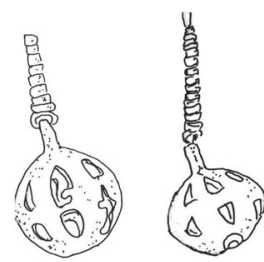

11
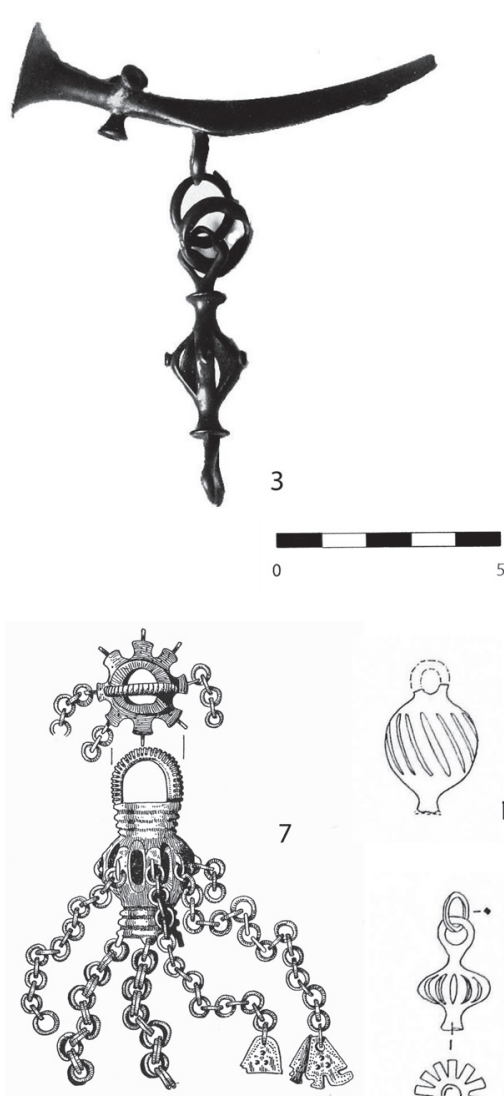

13

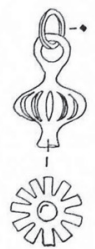

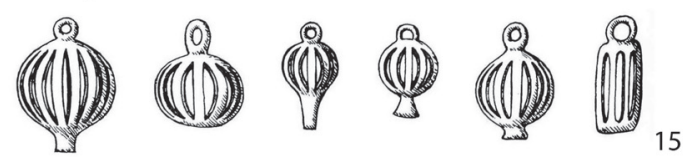

Abb. 1. Schlitzbommeln der späten Bronzezeit und frühen Eisenzeit

1 - Pécs (Ungarn); 2 - Ugra (Ungarn); 3 - Niederösterreich? (Österreich). Abbildung nach S. Gallus - T. Horvath 1939, kér 3; VI/3; XVIII/8. 4 - Dalj (Kroatien); 5 - Gajina pecina (Kroatien). Abbildung nach K. Vinski-Gasparini 1973, Tab. 119/6, Tab. 128/4-5. 6 - Most na Soci, Slowenien. Abbildung nach B. Teržan 1985, t. 44 F 519/8. 7 - Hallstatt (Österreich). Abbildung nach K. Kromer 1959, Taf. 149/1, Zeichnung W. Strasil. 8 - Südwestdeutschland. Abbildung nach A. Frey - C. Nickel - R. Köster 2009, 107, Aquarell L. Lindenschmidt. 9 - 10: Rusanovići und Iljak, Glasinac, Bosnien-Herzegovina. Abbildung nach J. Bouzek 2012, Fig. 6, 14 und 16. 11 - Bologna. (Italien). Zeichnung nach Foto B. M. Pomberger. 12 - Býčí-skála-Höhle (Tschechien). Abbildung nach H. Parzinger J. Nekvasil - F. E. Barth 1995, Taf. 20, 218a. 13-14 - Subingen (Schweiz). Abbildung nach W. Drack 1966, Abb. 12, 16 und 19, Maßstab 1:1. 15 - Bommeln der Trachtengruppe Moidons, Jura, Ostfrankreich. Abbildungen nach G. Wamser 1975, Taf. 4/1-6 (1, 2, 8 ohne Maßstab). 
(Niederkaina, Kr. Bautzen, Böderau, Kr. Riesa) und Polen (Wicina, pow. Lubsko). Die Funde, deren Form mit Typ 911/35120 nicht weiter beschrieben ist, datieren in die Phase Billendorf II (Buck 1979, 9, 142, 62).

Die Großzahl der mitteleuropäischen Stücke sind Grabbeigaben, Schmuckstücke, die im Brustbereich aufgefunden wurden. Dies wird auch für Funde in Griechenland berichtet $(K i$ lian-Dirlmeier 1979, 79-80). Einige Objekte wurden als Opfergaben in Heiligtümern der Artemis (Artemis Enodia-Heiligtum von Pherai) und der Athena (Athena Itonia-Heiligtum in Philia), dargebracht (Kilian-Dirlmeier 1979, 7879, 86-87, 99). Während in der jüngeren Phase der Hallstattzeit diese Art von Trachtschmuck in Mitteleuropa verschwindet, bleiben sie in Serbien bis ins 2. Jhdt. n. Chr. erhalten (KilianDirlmeier 1979, 80) (Abb. 1).

\subsection{Schellen der späten Urnenfelder- kultur und der Hallstattkultur}

Die Schellen der späten Urnenfelder- und frühen Hallstattkultur stehen klar in Verbindung mit der Erfindung geschlitzter Bommeln. Das Verbreitungsgebiet der Hallstattkultur reicht von der Donau im Osten bis zur Mündung der Flüsse Yonne und Aube in die Seine. Die in diesem Kulturraum gefundenen geschlitzten Bronzeschellen wurden wohl als apotropäischer Trachtschmuck getragen. Die derzeit als östlichste Funde bekannte, Schellen sind jene aus dem Hort von Simaság, Komitat Vas, Ungarn (Hampel 1896, Taf. 228, Abb. 1-2), die anhand des Tüllenbeiles frühestens in die Stufe HaB3/HaC datiert werden können (Pomberger 2016, 107). Amália Mozsolics stellt den Hortfund von Simaság ebenfalls in die Stufe HaB3 und ordnet ihn dem Depotfundhorizont Bükkszentlászló zu (Mozsolics 2000, 18, Abb. 3, 29, 74, Taf. 89,5). Die ca. 6,9 cm großen run- den Schellen sind mit zwei bzw. drei Eisenkugeln gefüllt. Der Grundton der mit zwei Kugeln gefüllten Schelle liegt bei $1900 \mathrm{~Hz}$, jener der zweiten bei $3500 \mathrm{~Hz}$ (Pomberger 2016, 108, 110, 251).

Aus Nordkroatien kennt man eine geschlitzte, leicht doppelkonische Schelle, die in Šarengrad 1897 in einem Hort entdeckt wurde. Sie ist gefüllt mit zwei Kügelchen und wird ins Ende der Phase V der kroatischen Urnenfelderkultur (HaB3) datiert (Vinski-Gasparini 1973, Sl. 7, 220, Tab. 131,3). Ein weiterer Fund einer geschlitzten Schelle stammt aus dem Grab 196 aus dem Gräberfeld Hallstatt-Hochtal, Oberösterreich. Dieses Idiophon, eingehängt an einer Pferdetrense, lag auf der rechten Seite des Brustkorbes der Bestatteten. Im Inneren dieses Idiophons befindet sich ein weißer Quarzstein als Rasselkörper (Kromer 1959, 68, Taf. 22,12; Pomberger 2016, 130, 257, Fototafel 13). Die in die Stufe HaC datierende Rassel (Hodson 1990, 67, 134) erklingt mit einem Grundton von 7838 $\mathrm{Hz}$ (Pomberger 2016, 139). Diese Schelle dürfte einerseits als Schmuck eines Pferdezaumzeuges gedient haben, andererseits als Halszierde von der Verstorbenen getragen worden sein. Eine Bronzeschelle mit kunstvoll in Wellenform ausgeführter Aufhängeöse darf aus Třtěno (Bezirk Lony, Ústecký kraj, Böhmen), genannt werden. Ihr kugelförmiger Körper enthält zwei Kugeln (Bouzek 2012, Fig. 6,15).

Bronzeschellen als Schmuckstücke/Trachtstücke wurden vor allem in der Schweiz in etlichen Gräbern aufgefunden. Hier sind folgende Gräber und Fundorte zu nennen: Im Grab des Hügels IV, Bussy/Valangin, Bezirk Val-De-Rutz, Kanton Neuenburg, lag die Schelle gefüllt mit einem Rasselkörper in Brusthöhe (Drack 1964, 24, 25, Taf. 8,11).

In einem Körpergrab im Bereich Les Cadolles in der Stadt Neuchâtel, Kanton Neuenburg, befand sich eine Schelle gefüllt mit zwei Kugeln (Drack 1964, 34, Taf. 12,8). 


\begin{tabular}{|c|c|c|c|c|c|c|}
\hline Schelle & Form & Rasselkörper & Maße H/Dm & Befund & Fundort/Land & Datierung \\
\hline Simaság I & kugelig & 3 Eisenkugeln & $6,9 \times 4,8 \mathrm{~cm}$ & Depot & $\begin{array}{l}\text { Simaság(Kom. } \\
\text { Vas, HU }\end{array}$ & $\mathrm{HaB3}$ \\
\hline Simaság II & kugelig & 2 Eisenkugeln & $6,9 \times 4,8 \mathrm{~cm}$ & Depot & $\begin{array}{l}\text { Simaság(Kom. } \\
\text { Vas, HU }\end{array}$ & HaB3 \\
\hline Šarengrad & $\begin{array}{l}\text { doppel } \\
\text { konisch }\end{array}$ & 2 Kugeln & $6,4 \times 4,4 \mathrm{~cm}$ & Depot & Šarengrad, HR & $\mathrm{HaB3}$ \\
\hline $\begin{array}{l}\text { Hallstatt- } \\
\text { Hochtal }\end{array}$ & kugelig & 1 Quarzstein & $6 \times 3,3 \mathrm{~cm}$ & $\begin{array}{l}\text { Grab 196, Frau, } \\
\text { an Trense } \\
\text { hängend, } \\
\text { Brustbereich }\end{array}$ & $\begin{array}{l}\text { Hallstatt, } \\
\text { Oberösterreich, } \\
\text { A }\end{array}$ & $\mathrm{HaC}$ \\
\hline Bex & kugelig & Stein & $5 \times 4 \mathrm{~cm}$ & ????? & Bex, VD, CH & $\mathrm{HaC} / \mathrm{HaD} 1$ \\
\hline Vauroux & kugelig & Kugel (?) & $4,8 \times 4 \mathrm{~cm}$ & $\begin{array}{l}\text { Grabhügel, } \\
\text { Körpergrab }\end{array}$ & Vauroux, VD, CH & $\mathrm{HaC} / \mathrm{HaD} 1$ \\
\hline Sion & $\begin{array}{l}\text { Kugelig mit } \\
\text { Fuß }\end{array}$ & 1 Stein (?) & $6,4 \times 4,6 \mathrm{~cm}$ & Streufund & Sion, VS, CH & $\mathrm{HaC} / \mathrm{HaD} 1$ \\
\hline Neuchâtel & $\begin{array}{l}\text { Kugelig mit } \\
\text { Fuß }\end{array}$ & 2 Kugeln & $4,4 \times 4 \mathrm{~cm}$ & $\begin{array}{l}\text { Grab, Körper- } \\
\text { grab }\end{array}$ & Neuchâtel, CH & $\mathrm{HaC} / \mathrm{HaD} 1$ \\
\hline Jouxtens & $\begin{array}{l}\text { Kugelig mit } \\
\text { Fuß }\end{array}$ & 1 Quarzstein & $5,6 \times 3,2 \mathrm{~cm}$ & $\begin{array}{l}\text { Grabhügel, } \\
\text { Körpergräber }\end{array}$ & Jouxtens, VD, CH & $\mathrm{HaC} / \mathrm{HaD} 1$ \\
\hline Bussy & $\begin{array}{l}\text { Kugelig mit } \\
\text { Fuß }\end{array}$ & 1 Stein (?) & $4,8 \times 3,2 \mathrm{~cm}$ & $\begin{array}{l}\text { Grabhügel IV, } \\
\text { Körpergrab, im } \\
\text { Brustbereich }\end{array}$ & $\begin{array}{l}\text { Bussy, Valangin, } \\
\mathrm{CH}\end{array}$ & $\mathrm{HaC} / \mathrm{HaD} 1$ \\
\hline Subingen 1 & $\begin{array}{l}\text { Kugelig mit } \\
\text { Fuß }\end{array}$ & 1 Kugel (?) & $4,8 \times 3,4 \mathrm{~cm}$ & $?$ & $\begin{array}{l}\text { Subingen, } \mathrm{SO}_{1} \\
\mathrm{CH}\end{array}$ & $\mathrm{HaC} / \mathrm{HaD} 1$ \\
\hline Subingen 2 & $\begin{array}{l}\text { Kugelig mit } \\
\text { Fuß }\end{array}$ & 1 Stein (?) & $4,2 \times 2,8 \mathrm{~cm}$ & $?$ & $\begin{array}{l}\text { Subingen, } \mathrm{SO} \text {, } \\
\mathrm{CH}\end{array}$ & $\mathrm{HaC} / \mathrm{HaD} 1$ \\
\hline Subingen 3 & $\begin{array}{l}\text { Kugelig mit } \\
\text { Fuß }\end{array}$ & 1 Stein (?) & $4,6 \times 3,8 \mathrm{~cm}$ & $?$ & $\begin{array}{l}\text { Subingen, } \mathrm{SO}, \\
\mathrm{CH}\end{array}$ & $\mathrm{HaC} / \mathrm{HaD} 1$ \\
\hline Wetzikon & kugelig & 1 Stein & $4,6 \times 3,4 \mathrm{~cm}$ & \begin{tabular}{|l|} 
Einzelfund an \\
tordiertem Stab
\end{tabular} & Wetzikon, $\mathrm{ZH}, \mathrm{CH}$ & $\mathrm{HaC} / \mathrm{HaD} 1$ \\
\hline Wetzikon & kugelig & 1 Stein & $4,6 \times 3,6 \mathrm{~cm}$ & $\begin{array}{l}\text { Einzelfund an } \\
\text { tordiertem Stab }\end{array}$ & Wetzikon, ZH, CH & $\mathrm{HaC} / \mathrm{HaD} 1$ \\
\hline Lyssach & $\begin{array}{l}\text { Kugelig mit } \\
\text { Fuß }\end{array}$ & 1 Bronzekugel & $5,2 \times 3,8 \mathrm{~cm}$ & $\begin{array}{l}\text { Grabhügel II, } \\
\text { Körpergrab }\end{array}$ & $\begin{array}{l}\text { Lyssach, Bern, } \\
\mathrm{CH}\end{array}$ & $\mathrm{HaC} / \mathrm{HaD} 1$ \\
\hline Třtěno & kugelig & 2 Kugeln & $5,6 \times 5 \mathrm{~cm}$ ?? & ? & $\begin{array}{l}\text { Třtěno, Ústecký } \\
\text { kraj, CZ }\end{array}$ & $\mathrm{HaC}$ ? \\
\hline Klein Jamno & $?$ & $?$ & $?$ & $\begin{array}{l}\text { Urnengrab, } \\
\text { Billendorfer } \\
\text { Gruppe }\end{array}$ & $\begin{array}{l}\text { Klein Jamno, } \\
\text { Kr. Forst, Bez. } \\
\text { Cottbus, D }\end{array}$ & $\mathrm{HaC}$ ? \\
\hline
\end{tabular}

Tab. 1. Tabelle der Schellen der Urnenfelder- und Hallstattkultur 

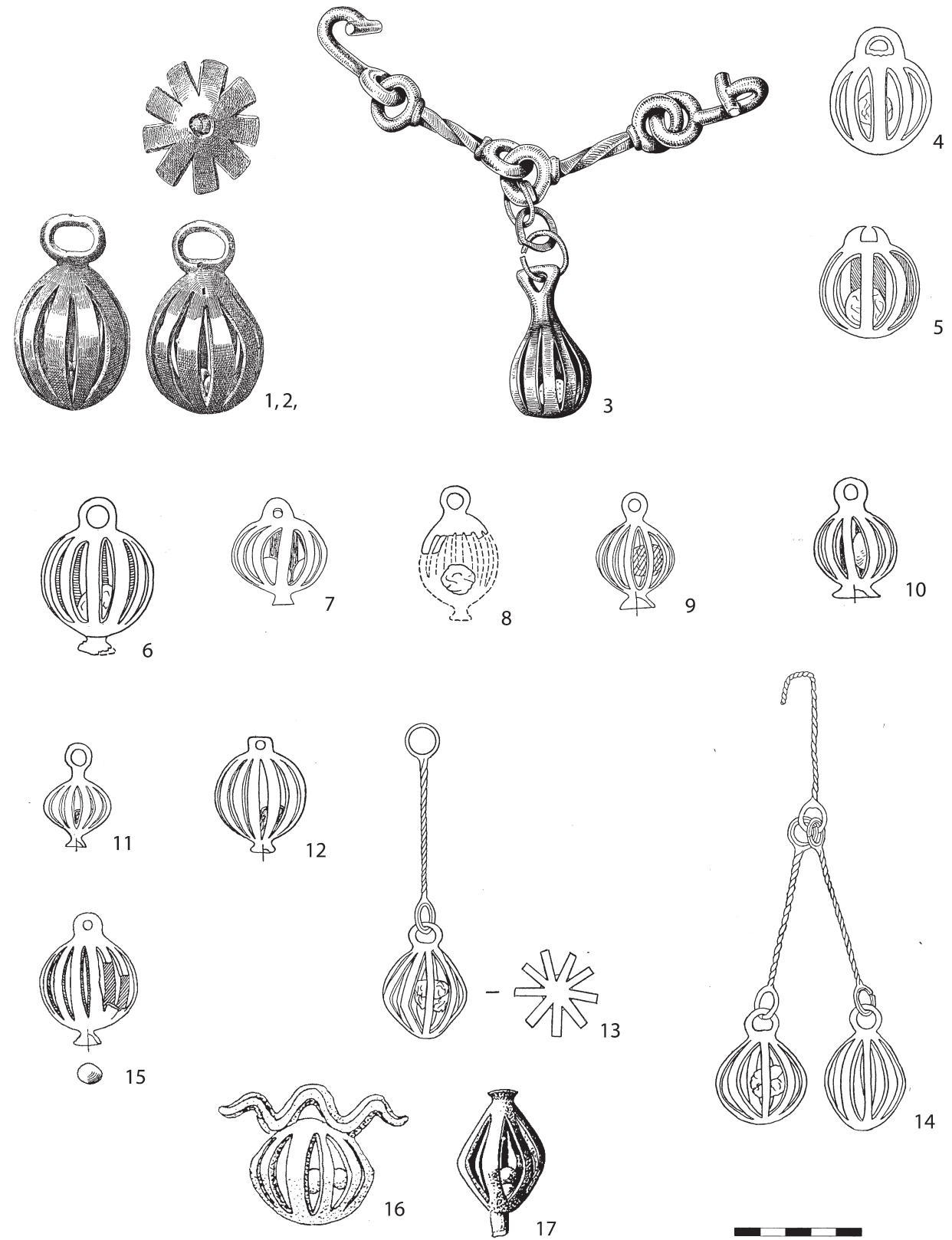

Abb. 2. Schellen der späten Urnenfelderkultur und Hallstattkultur

1, 2 - Simaság (Ungarn). Abbildungen nach J. Hampel 1896, Taf. 228/Abb. 1-2. 3 - Hallstatt (Österreich) Abbildungen nach K. Kromer 1959, 68, Taf. 22/12, Zeichnung W. Strasil. 4 - Bex; 5 - Vouroux, 6 - Sion, 7 - Neuchâtel, 8- Jouxtens, 9 - Bussy, 10-12 - Subingen, 13, 14 - Wetzikon, 15 - Lyssach (alle Schweiz). Abbildungen nach W. Drack 1966, 41, 42, Abb. 12 und 13. 16 - Trrtěno (Tschechien). Abbildungen nach J. Bouzek 2012, 544, Fig. 6/15; 17 - Šarengrad (Kroatien). Abbildungen nach K. Vinski-Gasparini 1971, Tab. 131, 3. 
Ein Grabhügel am Fuße des Jura in der Nähe von Vauroux/Bevaix, Kanton Neuenburg, barg eine weitere Schelle (Drack 1964, 36, Taf. 13,7). Ebenso ist eine Schelle aus Bex, Kanton Waadt (Drack 1964, 43, Taf. 18,1) zu nennen. In einem Tumulus im Wald Vernand de Blonay, JouxtensMezery, District Lausanne, Kanton Waadt, wurden vier Frauenbestattungen entdeckt, deren Grabbeigabe unter anderem eine Bronzeschelle war (Drack 1964, 48, 49, Taf. 21,10), (Abb. 3,1). Eine weitere Schelle wurde in Sion, Bezirk Sitten, Kanton Wallis aufgefunden (Drack 1964, 62, Taf. 28,8). Gleich drei Schellen mit Stempelabschluss („Füßchen“) stammen aus Subingen, Bezirk Wasseramt, Kanton Solothurn, gefüllt mit je einem Rasselkörper (Drack 1966, 40, Abb. 12/12, 17, 20). Zwei Einzelfunde stammen zuletzt aus Wetzikon, Kanton Zürich (Drack 1966, 41, Abb. 13/27, 28). Walter Drack hat 1966 alle geschlitzten Bommeln - mit oder ohne Rasselkörper - einer Typologie unterzogen (Drack 1966, 29-70, Abb. 12, 13), unterscheidet jedoch nicht zwischen Rassel und geschlitztem Anhänger. Anhand Dracks Typologie lassen sich die Schweizer Schellen grob in kugelige Typen (Typen Bex und Wetzikon), und Typen mit Füßchen (Typen Neuchâtel, Bofflens, Subingen II, Lyssach) einteilen. Meist sind sie mit nur einer Kugel gefüllt, außer einem Exemplar des Typs Neuchâtel, das zwei Kugeln beinhaltet. Die Typen Bex und Bofflens stellt Drack spätestens in die Stufe HaD1.

Schellen sind auch in Ostdeutschland in der jüngeren Bronzezeit/frühen Eisenzeit aus der Billendorfer Gruppe belegt, wie das Beispiel eines kleinen Funds $(1,5 \mathrm{~cm}$ mal $0,9 \mathrm{~cm})$ aus einem Urnengrab von Klein Jamno, Kr. Forst, Bez. Cottbus (Buck 1977, 1, 52) zeigt.

Um 600 B.C. - HaD1 - bricht die Hallstattkultur im pannonisch-ostalpinen Raum zusammen. Der Grund dürften kriegerische Einfälle der Skythen sein. Die befestigten Höhensiedlungen gehen in Flammen auf und werden aufgegeben (Teržan 1998, 520-521). Für die südostalpine Dolenjsko-Gruppe in Slowenien jedoch sind Kontakte zu Skythen belegt. So befand sich zum Beispiel in einem Grab mit Pfeilspitzen und Pferdegeschirr des Typs Szentes Vekerzug auch ein skythisches Glöckchen (Teržan 1998, 529, Taf. 10,5). Die Darstellung der Anreise zu einem Fest mit Pferd und Wagen (Eibner 2015) auf der Situla von Vače, Slowenien, wobei die Pferde mit je zwei Schellen geschmückt sind (Kastelic 1964, Taf. 5; Pomberger 2016, 130, Abb. 103), könnte mit der Übernahme skythischer Gepflogenheiten in Verbindung gebracht werden. Bei den Schellen selber dürfte es sich um größere runde Exemplare handeln (Abb. 3,2).

\subsection{Die Schellen der Skythen}

„... Ausgehend vom Nordschwarzmeerraum erreichen skythische Gruppen ebenso wie Teile der skythischen Sachkultur auch noch weiter westlich gelegene Gebiete ... “ (Parzinger 2004, 119). Es handelt sich bei diesen Gruppen um die sogenannten Agathyrsen, Träger der Vekerzug-Kultur, die sich im Laufe des 7. Jahrhunderts zunächst im Ostteil der Karpaten und später um 600 B.C. sich weiter westlich bis zur Donau ausbreiteten. Eine ihrer lokalen Vorgängergruppen ist die Mezőcsat-Kultur, die ebenfalls Verbindungen zum nördlichen Schwarzmeergebiet besaß (Parzinger 2004; Kemenczei 2007, 310-317).

$\mathrm{Zu}$ den kulturellen Hinterlassenschaften der skythisch geprägten Gruppen zählen bronzene Stangenbekrönungen mit einem als Schelle ausgebildeten Körper. Eine umfangreiche Arbeit über diese karpatischen Bronzerasseln hat Kornél Bakay 1971 mit seinem Werk „Scythian Rattles in the Carpathian Basin and their eastern Connections" vorgelegt (Bakay 1971). So kennt man sechs Schellen aus Gyöngyös, Kom. Heves, drei Stück aus Nagytarcsa, eine aus Aszód, beide Kom. Pest, 

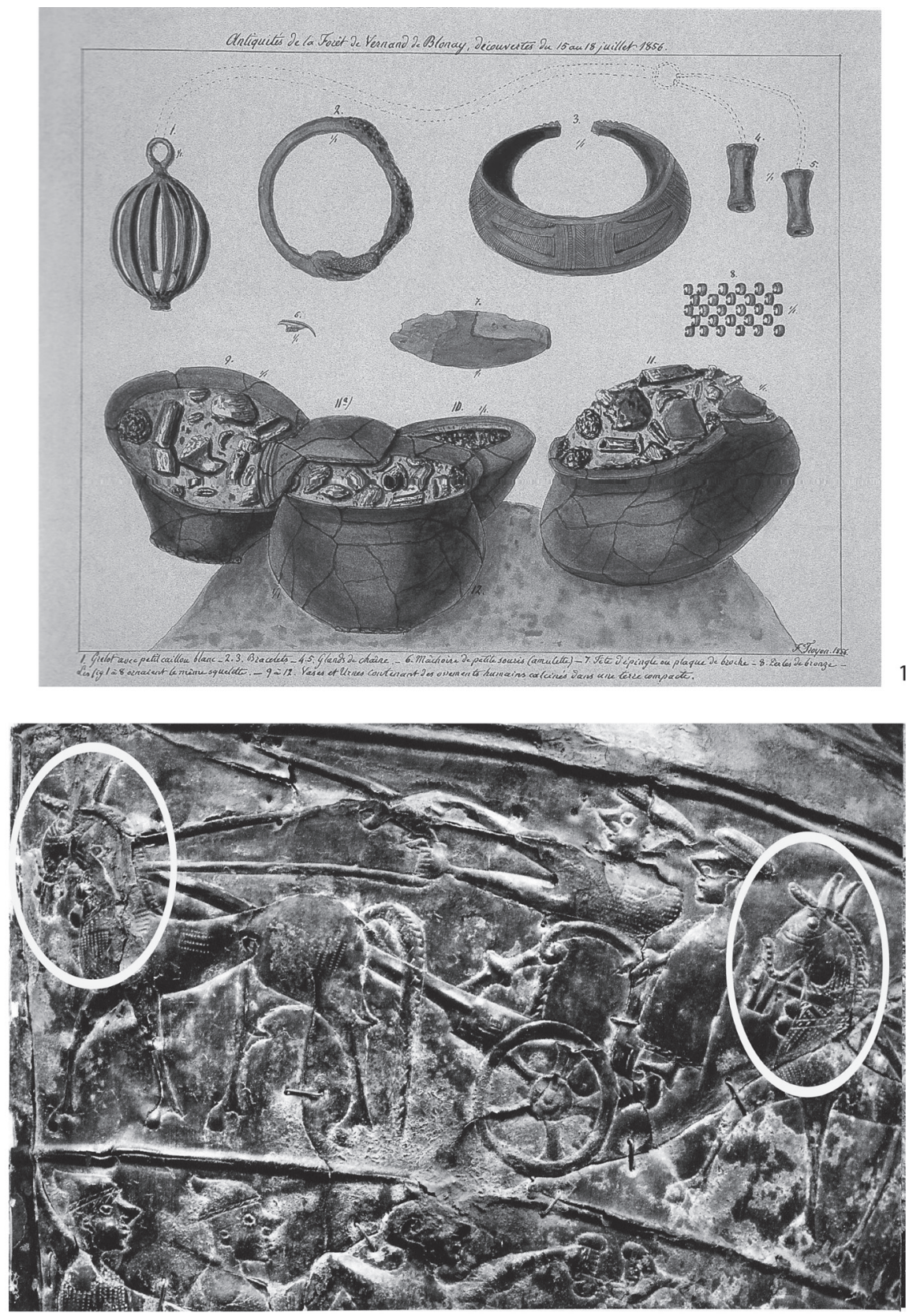

2

Abb. 3. Bilder mit Schellen

1 - Urnengrab aus Vernand de Blonay, Jouxtens-Mezery, Lausanne. Aquarelle von Frédéric Troyon. Abbildung nach F. Müller - G. Lüscher 2004, 21, Abb. 17.

2 - Anreise der Gäste zum Fest mit Pferd und Wagen, Fries der Situla von Vače (Slowenien). Abbildung nach J. Kastelic 1964, Taf. 5. 

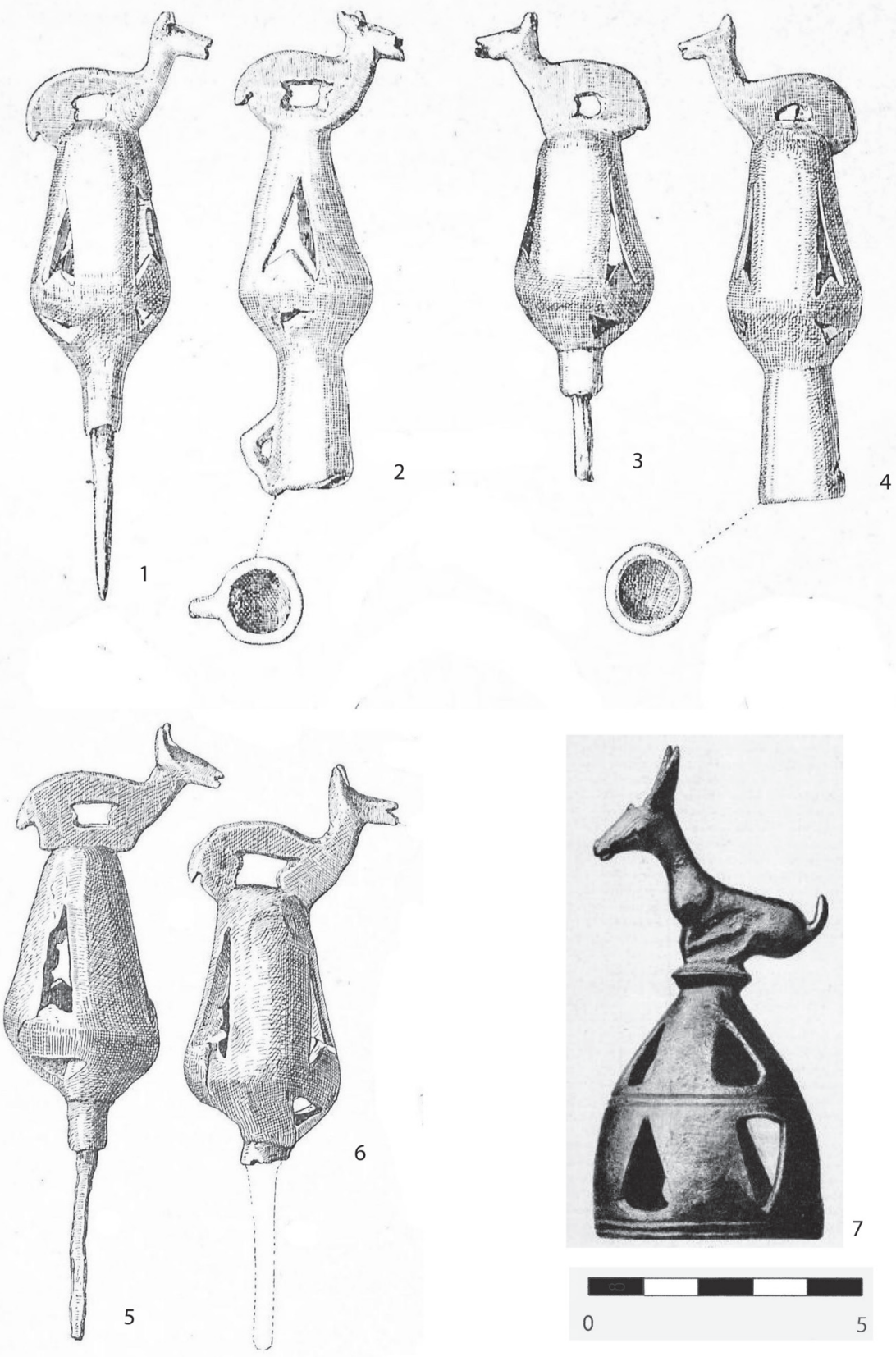

Abb. 4. Schellen der Skythen - Stangenbekrönungen in Ungarn

1, 2, 3, 4, 5, 6 - Gyöngyös (Ungarn). Abbildungen nach L. Márton 1908, I. Táb. 10-13, V. Táb. 1-2a; 7 - Gorneşti (Rumänien). Abbildung nach J. Hampel 1893, 403, 13. ábra. 
drei weitere aus Szurdockpüspöki, Kom. Nógrád sowie ursprünglich vier Idiophone aus Mihályfa, Kom. Veszprém. Die rumänischen Funde sind je eine Schelle aus Gyulafehérvár (Alba Julia), Somhíd (Nâadap) und Gorneşti, sowie vier weitere Stück aus Transsilvanien. Entdeckt wurden sie in Horten, Gräbern und als Streufunde. Ihre kunstvollen bronzenen Klangkörper sind doppelkonisch oder bienenkorbförmig ausgeführt und sitzen auf zylindrischen Sockeln auf. Ihr Mantel wird durch mehrere dreieckige oder karoähnliche Öffnungen durchbrochen. Eine Tierfigur - z. B. Hirsch, Rind, Vogel, Raubkatzen oder Pferd - krönt die Komposition aus Bronze. Die Gesamthöhe der Stangenbekrönungen beträgt zwischen 8,2-26 cm, die Höhen der Schellenkörper liegen zwischen 6,7-12,5 cm, ihre Durchmesser zwischen ca. 4,1-8,5 cm, ihr Gewicht beträgt zwischen 104-830g. Die Wandstärke wird mit 3-5,5 mm angegeben. Die Legierung der Bronze setzt sich aus 90\% Kupfer und 10\% Zinn zusammen. Hauptsächlich dienen Steinchen und Eisenkugeln als Rasselkörper. Einzig die Schelle aus Gyulafehérvár (Alba Julia) ist mit zwei Bronzekügelchen gefüllt. Beim Spiel der Gefäßrasseln erklingen Geräusche, deren Grundfrequenzen je nach Größe des Klangkörpers und Menge bzw. Material der Rasselkörper im Bereich von 1316-3597 Hz liegen (Bakay 1971, 63). Um die Idiophone zum Klingen zu bringen, ohne dabei zu ermüden, könnten die Stangen, auf denen die Instrumente steckten, 50-150 cm lang gewesen sein. Wie Kornél Bakay feststellen konnte, gleicht keine der in Ungarn entdeckten Schellen einer anderen, woraus zu schließen ist, dass für jedes Stück eine eigene Gussform entworfen und hergestellt werden musste. Die skythischen Metallidiophone im Karpatenbecken datieren in das 6./5. Jahrhundert B.C. (Abb. 4 und 5). Vergleichsfunde wurden im eigentlichen Siedlungsgebiet der skythischen Reiterkrieger im nordpontischen Raum - hier vor allem an Don, Sala, Dnjestr, Sula, im Kuban und bei Kiev, entdeckt (Bakay 1971). Ihr eigentlicher Gebrauch ist nach wie vor umstritten, könnte aber auch mit Schamanismus in Verbindung gebracht werden (Parzinger 2004, 122). Bakay ortet die Vorläufer skythischer Schellen Ende des 3. Jahrtausends in Anatolien, von wo aus sie nach Mesopotamien, Assyrien, Urartu und in Folge im 14./13. Jahrhundert B.C. ins Kaukasusgebiet gelangten. Die frühesten Exemplare skythischer Schellen stammen aus der Kubanregion (Bakay 1971, 101-108). Mit den skythischen Völkern verschwinden offensichtlich in Südosteuropa im 3. Jahrhundert B.C. Nachweise für Metallrasseln. Sie sind aber weiterhin für die jüngere Eisenzeit der Steppenkulturen, z. B. in der Ol'ga-Kultur in Primor'e in Ostsibirien, jüngere Eisenzeit, belegt (Parzinger 2006, Abb. 250,23). Mit dem Eindringen der Awaren im 6. Jahrhundert nach Christus gelangten Schellen wieder nach Europa.

\subsection{Schellen aus dem spätbronze- eisenzeitlichen Irland}

Im Irland der ausgehenden Bronzezeit/beginnenden Eisenzeit erreichte die Metallurgie eine bemerkenswerte hohe Qualität in der Herstellung von Guss und Blech. So wurden nicht nur Hörner gegossen, sondern handtellergroße Schellen - irisch: Crotals - aus Bronze erzeugt, in deren Inneren sich eine Kugel oder ein Steinchen als Rasselkörper befindet. 48 solcher Schellen wurden im Hort von Dowris, Dowrys/Birr, County Offyle ergraben. Das Depot wurde in den 1820er Jahren beim Erdäpfelstechen entdeckten und enthielt mehr als 200 Objekte, wie Lanzenspitzen, Tüllenbeile, Schwerter, Situlen, Meißel und Messer und 26 Hörnern. Die Funde datieren in die sogenannte Dowris Phase (9./8. 

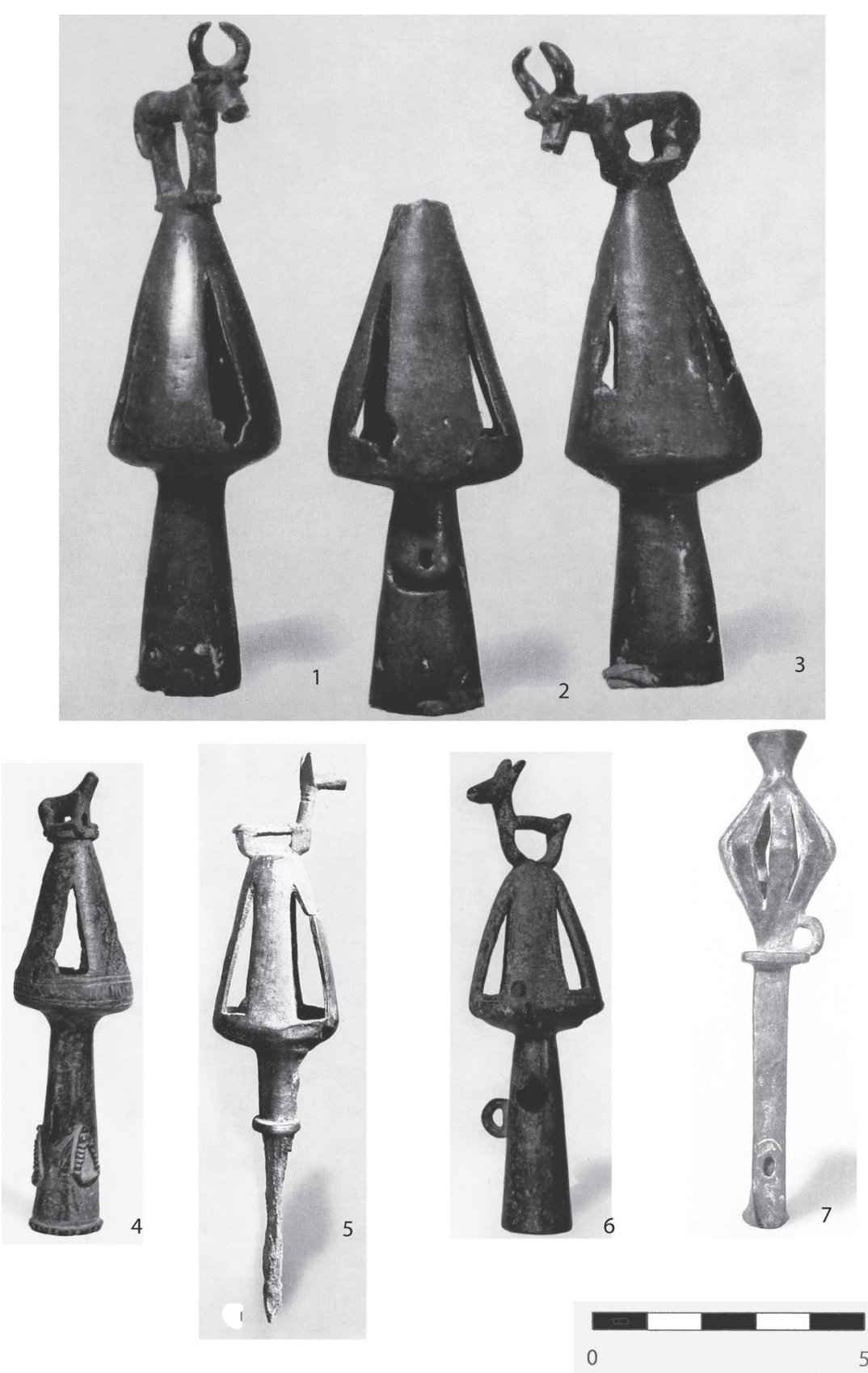

Abb. 5. Schellen der Skythen - Stangenbekrönungen in Ungarn.

1, 2, 3 - Nagytarcsa (Ungarn), 4 - Szurdokpüspöki (Ungarn), 5 - Mihályfa (Ungarn), 6 - Nâdap (Somhíd) (Rumänien?), 7 - Gyulafehervár (Alba lulia) (Rumänien). Abbildungen nach K. Bakay 1971, PI. l; PI. VIII; PI. XII; PI. XI; PI. X). 

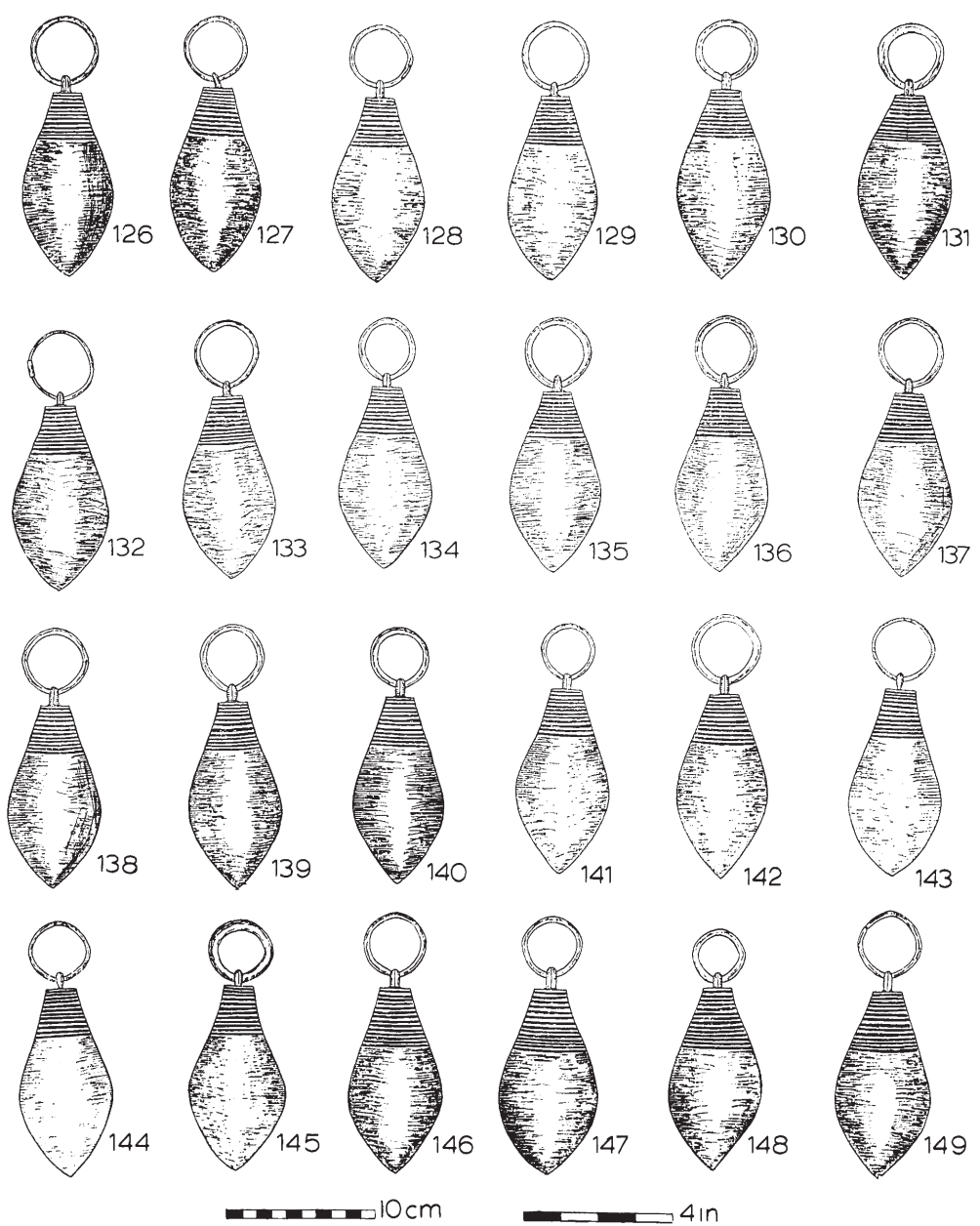

Abb. 6. Schellen aus Irland - Hoard of Dowris. Abbildungen nach G. Eogan 1983, Tab. 77.

Jhdt. B.C. - 600 B.C.). Die Schellen ähneln in ihren Formen Birnen und etwas ovalen Kugeln, deren oberes Ende mit umlaufenden Rillen verziert ist. Eogan teilt sie in Typen mit 14 Rillen (20 Stück), solchen mit 12 Rillen (20 Stück) und Mischformen (8 Stück). Sie messen ca. $12 \mathrm{~cm}$ in der Höhe und haben Durchmesser von 6,3-6,8 cm (Eogan 1983, XX, 7, 8, 136-140, 293, 294) (Abb. 6).
Als Abschluss sitzt ein Ring, an dem man das Instrument aufhängen, oder durch den man den Zeigefinger führt, um das Idiophon zum Spielen halten zu können (O’Dwyer 2015, 57, Fig. 33). Frequenzanalysen liegen nicht vor. Die irischen Schellen unterscheiden sich in Form und Gusstechnik völlig von jenen aus dem Festland Europas, daher dürfte es sich um eine eigene Erfindung handeln. 

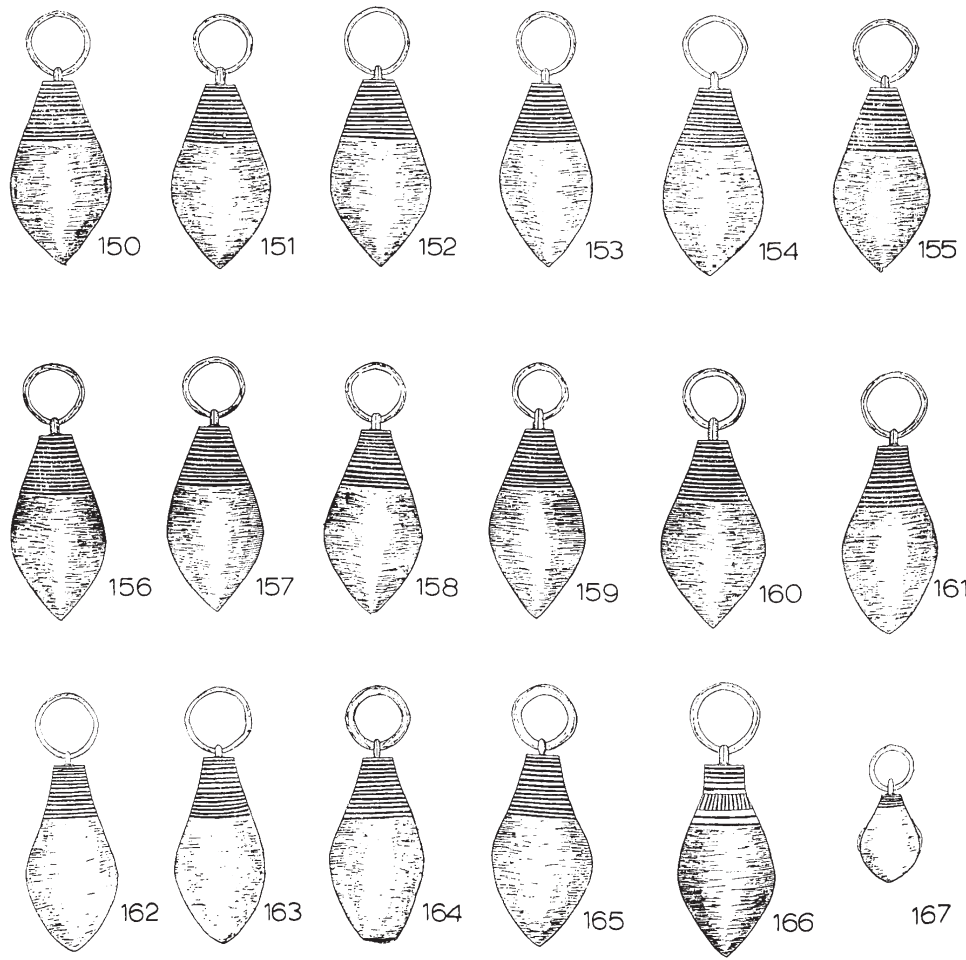

167
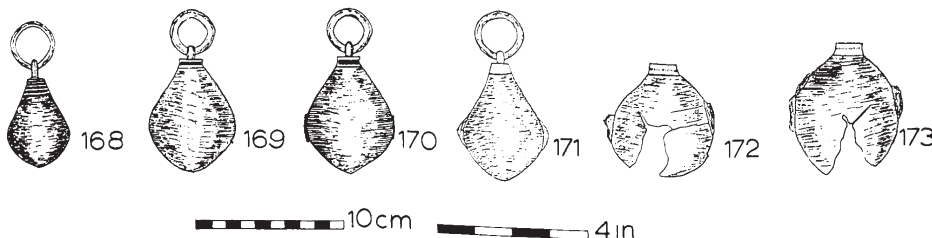

$4 \mathrm{in}$

Abb. 7. Schellen aus Irland - Hoard of Dowris. Abbildungen nach G. Eogan 1983, Tab. 78.

\section{Schlussbetrachtung und Ausblick}

Die Idee Rasseln aus Bronze zu gießen entstand eindeutig in der Zeit des Übergangs der Spätbronzezeit zur frühen Eisenzeit, wahrscheinlich durch vermehrte Kulturkontakte zwischen dem Kaukasus, Zentraleuropa und dem Schwarzmeergebiet. Schlitzbommeln kommen vom Iran (Luristan) über den Westbalkan, Un- garn bis Tschechien, Österreich, Schweiz und Ostfrankreich in den Phasen HaB3-HaD1 vor, werden vorwiegend als Anhänger um den Hals von Frauen getragen, sind aber auch fallweise als Details reicher Ziergehänge, wie in Ostfrankreich, Mähren und Griechenland, belegt. Aus diesen Schlitzbommeln entstanden sehr wahrscheinlich zeitgleich die ersten Schellen: Schlitzbommeln gefüllt mit Steinchen oder Kügelchen, die wiederum von Ungarn bis in 


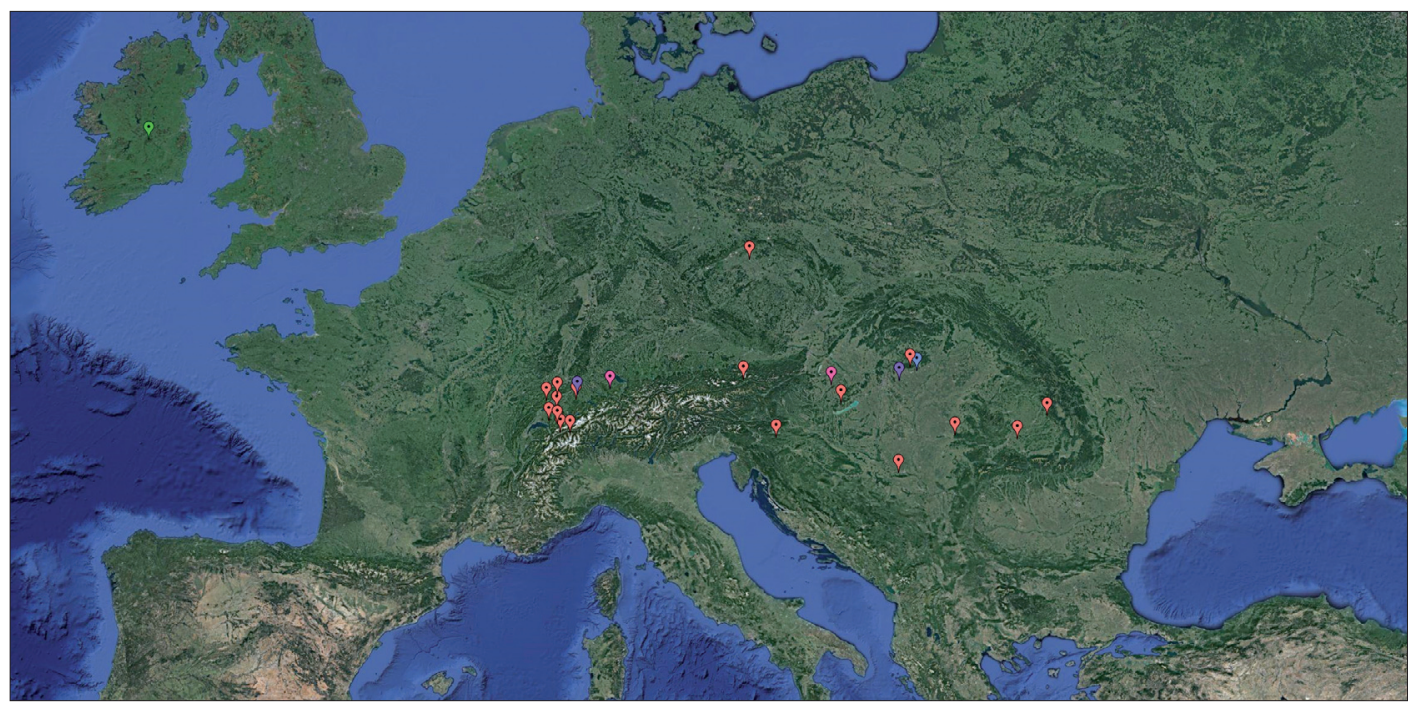

Abb. 8. Verbreitungskarte der Schellen zwischen Irland und dem Karpatenbecken von 900 - 300 B.C. (Erstellung: Peter Stadler, GoogleMaps).

die Schweiz in den Phasen HaB3 - HaD1 auftreten. Auch sie werden hauptsächlich als apotropäischer Halsschmuck von Frauen getragen. Eher selten sind Schlitzbommeln und Schellen im Kontext mit Pferdezaumzeug belegt. Skythische Schellen als Stangenbekrönungen sind offensichtlich jünger als die Schellen der ausgehenden Urnenfelderkultur/beginnenden Eisenzeit, da die ersten Funde in Ungarn um 600 B.C. (HaD1) datieren und die nordpontischen gar noch jünger sind. Es drängt sich der Verdacht auf, dass skythische und mitteleuropäische Schellen wohl die gleichen Wurzeln haben, die im Iran und dem Kaukasus zu suchen sind. Die Schellen Irlands hingegen sind offensichtlich eine lokale Erfindung, da ihr Aussehen und ihre Größe sich deutlich unterscheiden von jenen Mittel-und Osteuropas. Erklingen die geschlitzten urnenfelder-hallstattzeitlichen Schellen ab Frequenzen von ca. $1900 \mathrm{~Hz}$ aufwärts, so besitzen die skythischen
Stücke Grundtöne ab ca. $1300 \mathrm{~Hz}$. Von den irischen Bronzerasseln liegen keine Frequenzmessungen vor. Nach 600 B.C. gibt es keine Schellen mehr in Irland und ebenso geraten sie in Mitteleuropa in Vergessenheit. Abbildung 8 zeigt die Verbreitung der Schellen inclusive der skythischen zwischen 900 - 300 B.C. im Gebiet Irland bis zum Karpatenbogen (Abb. 8). Die Skythen und in Folge weitere Steppenvölker und Völker Asiens jedoch bis hin zum östlichen Ende des Kontinents dürften diese bronzenen Idiophone in Verwendung gehabt haben. Erst mit dem Eindringen der Awaren kommen sie wieder zurück nach Europa, wo sie im Mittelalter zum begehrten Trachtschmuck wurden und bis heute in Gebrauch sind. Eine künftige Aufgabe wird es sein, die Geschichte der Schellen, jene ihrer Bedeutungen und die ihres Klanges in Eurasien zu erforschen. Mein Dank geht an Peter Stadler für die Erstellung der Verbreitungskarte und an GoogleMaps. 


\section{Literaturverzeichnis}

Bakay, K. 1971: Scythian Rattles in the Carpathian Basin and their Eastern Connections. AmsterdamBudapest.

Behrends, Chr. 2010: Klänge der Bronzezeit. Musikarchäologische Studien über bronzezeitliche Hortfunde Mitteldeutschlands, Universitätsforschungen zur prähistorischen Archäologie Band 187. Bonn.

Bouzek, J. 1974: Macedonian Bronzes: their origins, distribution and relation to other cultural groups of the Early Iron Age, Památky archeologické LXV, 278-341.

Bouzek, J. 2012: Central Europe and Caucasus in the Early Iron Age. In: Blajer, W. (Hrsg.): Peregrinationes archaeologicae in Asia et Europa Joanni Chochorowski dedicatae. Krakau.

Buchvaldek, M. - Lippert, A. - Kosnar, L. (Hrsg.) 2007: Atlas zur prähistorischen Archäologie Europas. Prag.

Buck, D.-W. 1977: Die Billendorfer Gruppe, Teil 1, Katalog Bezirke Potsdam, Frankfurt und Cottbus der DDR und Westberlin. Veröffentlichungen des Museums für Ur- und Frühgeschichte Potsdam 11. Berlin.

Buck, D. -W. 1979: Die Billendorfer Gruppe, Teil 2, Text, Veröffentlichungen des Museums für Urund Frühgeschichte Potsdam 13. Berlin.

Clodoré T. 2002: Préhistoire de la musique. Sons et instruments de musique des âges du Bronze et du Fer en France. Nemours.

Dietz U. L. 1998: Spätbronze- und früheisenzeitliche Trensen im Nordschwarzmeergebiet und am Nordkaukasus. Prähistorische Bronzefunde Abteilung XVI, Band 5. Stuttgart.

Drack, W. 1958: Die ältere Eisenzeit der Schweiz, Materialhefte zur Ur- und Frühgeschichte der Schweiz 1. Basel.

Drack, W. 1962: Die ältere Eisenzeit der Schweiz, Materialhefte zur Ur- und Frühgeschichte der Schweiz 3. Basel.

Drack, W. 1964: Die ältere Eisenzeit der Schweiz, Materialhefte zur Ur- und Frühgeschichte der Schweiz 4. Basel.

Drack, W. 1966: Anhängeschmuck der Hallstattzeit aus dem schweizerischem Mittelland und Jura,
Jahrbuch der Schweizerischen Gesellschaft für Ur- und Frühgeschichte, Band 53, 29-70.

Eibner, A. - Forstenpointner, G. 2009: Zu Fragen der eisenzeitlichen Pferdebeschirrung und Deichselbefestigung nach den Bildbelegen, Mitteilungen der Anthropologischen Gesellschaft in Wien 139 (= Festschrift Fritz Eckart Barth zum 70. Geburtstag), 217-230.

Eibner, A. 2015: Feste und Rituale in der Situlenkunst, Mitteilungen der Anthropologischen Gesellschaft in Wien, CXLV. Band, 55-84.

Eogan, G. 1983: The hoards of the Irish later Bronze Age. University College Dublin, Dublin.

Frey, A. - Nickel, C. - Köster, R. 2009: Ein Bronzegehänge aus Slowenien (6. Jahrhundert v. Chr.), Jahrbuch des Römisch-Germanischen Zentralmuseum Mainz 56/3.

Hänsel, B. - Machnik, J. 1998: Das Karpatenbecken und die osteuropäische Steppe: Nomadenbewegungen und Kulturaustausch in den vorchristlichen Metallzeiten (4000-500 v. Chr.). Südosteuropa-Schriften 20, Rahden-Westfalen.

Gallus, S. - Horvath, T. 1939: Un people cavalier préscythique en Hongrie. Trouvaille archéologiques du premier Âge du Fer et leurs relations avec l'Eurasie. Dissertationes Pannonicae Serie II.9, Budapest.

Hampel, J. 1896: A bronzkor emlékei Magyarhonban. Budapest.

Hampel, J. 1893: Skythiai emlékek Magyarországban, Archaeologiai értesító XIII (1893), 385-407.

Hodson, F. R. 1990: Hallstatt - the Ramsauer graves: quantification and analysis. Monographien / Römisch-Germanisches Zentralmuseum Mainz, 16, Bonn

Hornbostel, E. M. - Sachs, C. 1914: Systematik der Musikinstrumente. Ein Versuch, Zeitschrift für Ethnologie 46, 553-590.

Kastelic, J. 1964: Situlenkunst. Meisterschöpfungen prähistorischer Bronzearbeit. Wien-München.

Kemenczei, T. 2007: Denkmäler skythisch geprägter Eliten im Donau-Theiß-Gebiet. In: Menghin, W. Parzinger, H. - Nagler, A. - Nawroth, M. (Hrsg.) Im Zeichen des goldenen Greifen. Königsgräber der Skythen. Deutsches Archäologisches Institut, München, 310-317.

Kienlin T. L. 2007: Von den Schmieden der Beile: $\mathrm{Zu}$ Verbreitung und Angleichung metallurgischen 
Wissens im Verlauf der Frühbronzezeit, Praehistorische Zeitschrift 82, 1-22.

Kilian-Dirlmeier, I. 1979: Anhänger in Griechenland von der mykenischen bis zur spätgeometrischen Zeit (Griechisches Festland, Ionische Inseln, dazu Albanien und Jugoslawisch Makedonien). Prähistorische Bronzefunde XI, 2, München.

Kromer, K. 1959: Das Gräberfeld von Hallstatt. Florenz.

Márton, L. 1908: Skytha sirleletek Gyöngyösről, Archaeologiai értesítő 28 (1908), 37-54.

Moszolics, A. 2000: Bronzefunde aus Ungarn. Depotfundhorizonte Hajdúböszörmény, Románd, und Bükkszéntlászló. Prähistorische Archäologie in Südosteuropa, 17, Kiel.

Müller, F. - Kaenel, G. - Lüscher, G. (Hrsg.) 1999: Eisenzeit - Age du Fer - Età del Ferro. Die Schweiz vom Paläolithikum bis zum frühen Mittelalter IV.

Müller, F. - Lüscher, G. 2004: Die Kelten in der Schweiz. Stuttgart.

Müller-Karpe, H. 1959: Beiträge zur Chronologie der Urnenfelderzeit nördlich und südlich der Alpen. Römisch-Germanische Kommission 22, Berlin.

O'Dwyer, S. 2015: Ancient music and instruments of Ireland and Britain: the story of a distinctive musical culture during the Stone Age, Bronze Age and Iron Ages of North Western Europe: harmonic flutes, horns, great Celtic trumpets: music archaeology in performance, parade, legend, healing, fanfare and war. North Charleston.

Parzinger, H. - Nekvasil, J. - Barth, F. E. 1995: Die Býči-Skala-Höhle. Ein hallstattzeitlicher Höhlenopferplatz in Mähren. Deutsches Archäologisches Institut, Römisch-Germanische Kommission 54, Main am Rhein.

Parzinger, H. 2004: Die Skythen. München.

Parzinger, H. 2006: Die frühen Völker Eurasiens. Vom Neolithikum bis zum Mittelalter. München.

Menghin, W. - Parzinger, H. - Nagler, A. - Nawroth, M. 2007: Im Zeichen des goldenen Greifen. Königsgräber der Skythen. Deutsches Archäologisches Institut, München.
Pomberger, B. M. 2016: Wiederentdeckte Klänge. Musikinstrumente und Klangobjekte vom Neolithikum bis zur Römische Kaiserzeit im mittleren Donauraum. Bonn.

Poppi, L. K. - Neri, D. 2015: Donne dell'Etruria padana dall' VIII al VII secolo a.C. tra gestione domestica et produzzione artigianale. Firenze.

Семенов, В. 2015: Искусство варварских племен. Санкт-Петерсбург.

Šolle, M. 1947/48: Halštatský hromadný nález z Ratají nad Sázavou, Památky archaeologické XLIII, 102-104.

Teržan, B. - Lo Schiavo, F. - Trampuž-Orel, N. 1984: Most na Soči (S. Lucia) II, 2. Szombathyjeva izkopavanja $=$ Die Ausgrabungen von J. Szombathy, Tafelband. Katalogi in monografije 23/2, Ljubljana.

Teržan, B. - Lo Schiavo, F. - Trampuž-Orel, N. 1985: Most na Soči (S. Lucia) II, 2. Szombathyjeva izkopavanja = Die Ausgrabungen von J. Szombathy, Text. Katalogi in monografije 23/1, Ljubljana.

Teržan, B. - Trampuž, N. 1973: Rispevek h kronologiji svetolucijske skupine, Arheološki vestnik 24, 416-460.

Teržan, B. 1998: Auswirkungen des skythischen geprägten Kulturkreises auf die hallstattzeitlichen Kulturgruppen Pannoniens und des Ostalpenraumes. In: Hänsel, B. - Machnik, J. (Hrsg.): Das Karpatenbecken und die osteuropäische Steppe. Nomadenbewegungen und Kulturaustausch in den vorchristlichen Metallzeiten (4000-500 v. Chr.). Südosteuropa-Schriften 20, Prähistorische Archäologie in Südosteuropa 12, Rahden-Westfalen, 511-560.

Tovoli, S. 1989: Il Sepulcro Villanoviano Benacci Caprara di Bologna. Bologna.

Vinski-Gasparini, K. 1973: Kultura polja sa žarma u sjevenernoj Hrvatskj (Die Urnenfelderkultur in Nordkroatien). Zadar.

Wamser, G. 1975: Zur Hallstattkultur in Ostfrankreich. Die Fundgruppen im Jura und Burgund, Bericht der Römisch Germanischen Kommission 56, 1-178. 


\section{Bronzebommeln und Schellen - eine klangliche Errungenschaft aus der Spätbronze- und frühen Eisenzeit}

Kannte man in der Urgeschichte Europas bis zum Ende des zweiten Jahrtausends Gefäßrasseln aus gebranntem Ton, Holz und getrockneten Früchten, so kommen im 10./9 Jhdt. v. Chr., - der auslaufenden Bronzezeit und der beginnenden Eisenzeit - geschlitzte Bommeln in verschiedensten Formen vom Kaukasus und Nordiran, über den Balkan und Mitteleuropa bis nach Ostfrankreich als Anhänger vor (Bouzek 2012; Kilian Dirlmeier 1979; Parzinger Nekvasil - Barth 1995), die vorwiegend von Frauen um den Hals getragen werden. Diese Pendelanhänger, gefüllt mit Steinchen oder kleinen Metallkugeln, ergeben die ersten metallenen Gefäßrasseln, Schellen genannt, und bereichern die Vielfalt der Idiophone mit ihrem "Glockenklang“, der auch als apotropäisch gilt. Selten sind sie Bestandteil des Pferdegeschirrs. Funde gibt es aus Ungarn, Kroa- tien, Österreich und zahlreicher in der Schweiz (Drack 1964, Drack 1966; Hampel 1896; Kromer 1959; Vinski-Gasparini 1973). Die jüngsten Stücke datieren spätestens in die Phase HaD1. Mit dem Eindringen der skythischen Agathyrsen ins Karpatenbecken bis zur Donau, den Trägern der Vekerzug-Kultur, verschwinden die Schellen der Hallstattkultur in Mitteleuropa. Die Reiternomaden jedoch führen Schellen mit durchbrochenen Resonanzkörpern als Stangenbekrönungen mit sich (Bakay 1971) und tradieren diese neue Erfindung weiter in Eurasien. Die Grundfrequenzen der mitteleuropäischen und skythischen Schellen liegen je nach Größe von 1300 $\mathrm{Hz}$ aufwärts (Pomberger 2016). Eine eigene Innovation jedoch stellen die spätbronzezeitlichen irischen Schellen dar, die nur im Hortfund von Dowris, Mittelirland, belegt sind (Eogan 1983). 


\section{Dr. Beate Maria Pomberger}

- Universität Wien - Institut für Urgeschichte und historische Archäologie

Franz-Klein-Gasse 1, A - 1190 Wien, Österreich beatemaria.pomberger@gmail.com 